\title{
INTERACTION BETWEEN MAGNETIC LAYERS IN STRUCTURES WITH NARROW-GAP IV-VI SEMICONDUCTORS
}

\author{
V.K. Dugaev ${ }^{a}$, V.I. LITvinov ${ }^{a *}$, W. Dobrowolski ${ }^{b}$ And T. Story ${ }^{b}$ \\ ${ }^{a}$ Chernovtsy Department of the Institute of Materials Science Problems \\ Ukrainian Academy of Sciences, Vilde 5, 274001 Chernovtsy, Ukraine \\ ${ }^{b}$ Institute of Physics, Polish Academy of Sciences \\ Al. Lotników 32/46, 02-668 Warsaw, Poland
}

The results of calculation of the indirect exchange interaction between magnetic layers are presented for the case of a structure with narrow-gap semiconducting IV-VI quantum well. The main mechanism is a magnetic polarization of the size-quantized electrons and holes inside the well. This type of interaction is suggested for the explanation of recent experiments on $\mathrm{EuS} / \mathrm{PbS}$ structures.

PACS numbers: 75.70.Cn, 75.50.Pp, 75.70.Ak

The problem of interaction between magnetic layers separated by a non-magnetic material (metal or semiconductor) is becoming important in connection with the recent advances of microtechnology and a search of new device applications. There is a large number of theoretical papers on the coupling between magnetically ordered layers separated by a non-magnetic metal. Several models have been proposed. Despite of different approaches to the problem, the main idea is a transfer of the magnetic polarization from one magnetic layer to another via free carriers of the non-magnetic metal. In other words, the Ruderman-Kittel-KasuyaYoshida (RKKY) mechanism has been always presented in some form. A review of theoretical works has been given in articles [1-3] (see also a discussion in [4]).

Here we consider a system consisting of a IV-VI based narrow-gap semiconducting quantum well (QW) between two magnetic layers. An example of such system is a EuS/PbS/EuS sandwich structure. The main peculiarity of this structure is that the electron energy spectrum of the material inside the well is of narrow-gap-semiconducting type with interband coupling which includes strong spin-orbit interaction. The quantization of the energy spectrum in a IV-VI based quantum well has been considered in [5].

It was shown in a recent experimental work [6] that in superlattices consisting of EuS/PbS/EuS layers, a ferromagnetic phase transition takes place with the

*Present address: Center for Quantum Devices, Northwestern University, Evanston IL 60208, USA. 
Curie temperature depending on the number of layers. The proposed explanation of this dependence was a variation of the critical temperature with the effective number of nearest neighbors (from one neighbor in $z$-direction for two magnetic layers, to two neighbors for infinity number). The energy scale of the magnetic phase transition in the whole volume is, most likely, the interaction between magnetic layers (EuS) mediated by electrons and holes inside the quantum well $(\mathrm{PbS})$.

Here we present the results of our calculation of the interaction between magnetic layers using a method developed in $[7,8]$ for the coupling between separated magnetic impurities in a quantum well of narrow-gap IV-VI semiconductors. The main assumption is that the interaction is due to magnetic polarization of electron-hole system without real carriers (an analogue of the BloembergenRowland mechanism [9]). Such an assumption is in good accordance with the conditions of the experiment [6]. We assume the energy model of this system to be of rectangular form, as presented in Fig. 1. It means that we neglect possible asymmetry of the energy spectrum with respect to the electron-hole reflection (as it was suggested in [10]).

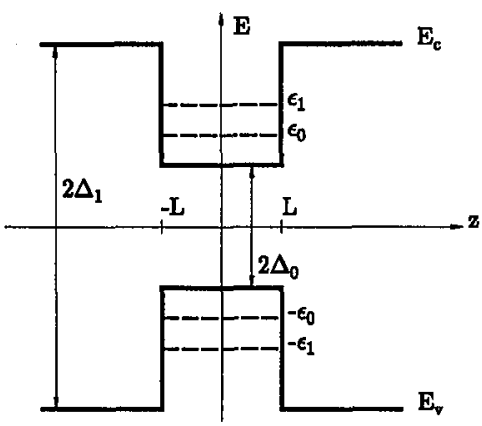

Fig. 1. The model of the quantum well.

The interaction energy of two magnetic impurities can be calculated as a coupling of one of them with a magnetic density created by another magnetic center. It was shown [8] that any magnetic impurity inside the symmetrical quantum well (see Fig. 1) can be characterized by its position $x$ along the well, and two coupling constants, $J_{\mathrm{s}}(z)$ and $J_{\mathrm{a}}(z)$, which are associated with the coupling to symmetric and antisymmetric parts of magnetic density, and $z$ is the impurity coordinate in direction perpendicular to the plane.

Using the formulae describing the coupling of two separated magnetic impurities in the quantum well of IV-VI narrow-gap semiconductor $[7,8]$, we can calculate the interaction between a magnetic moment situated at one side of the quantum well $(z=L)$ and all magnetic moments situated at the other side of the well $(z=-L)$. This quantity gives us the interaction energy per spin at the outer side of the magnetic layer. It determines the temperature of magnetic ordering of neighboring layers.

In the case of magnetic moments directed perpendicular to the well, making use of $[7,8]$ and after integrating over $x$, we get (some details of calculations can 
be found in [11])

where

$$
E_{\text {int }}^{t}=-\frac{2 N_{v} n_{i} E_{m} J^{2} N^{4}}{v^{2}} \exp \left(-4 \kappa_{1} L\right)\left(\kappa_{0} d\right)^{2}\left[1+\cot ^{2}\left(2 \kappa_{0} L\right)\right],
$$

$$
\begin{aligned}
& \kappa_{0}^{2}=\frac{1}{v}\left(\varepsilon_{0}^{2}-\Delta_{0}^{2}\right), \quad \kappa_{1}^{2}=\frac{1}{v}\left(\Delta_{1}^{2}-\varepsilon_{0}^{2}\right), \\
& N^{2}=\frac{\exp \left(2 \kappa_{1} L\right)}{2}\left[\frac{1}{\kappa_{1}}+\frac{2 L}{\sin ^{2}\left(2 \kappa_{0} L\right)}-\frac{\cot \left(2 \kappa_{0} L\right)}{\kappa_{0}}\right]^{-1},
\end{aligned}
$$

$n_{i}$ is the surface density of magnetic moments, $N_{v}$ is the number of valleys (four in $\mathrm{PbS}$ ), $J$ is the "bare" coupling constant of a bulk material, $d$ is the range of magnetic-impurity-electron coupling (of the order of lattice constant $a_{0}$ ), $E_{m}$ is the high-energy cut-off for the linear spectra in IV-VI semiconductor (about $0.5 \mathrm{eV}$ for $\mathrm{PbS}$ [12]), and $v$ is the interband coupling. In the case of moments directed along the QW plane, the calculation gives us $E_{\text {int }}^{l}=E_{\text {int }}^{t} / 4$.

In both cases the coupling is ferromagnetic. For numerical estimations we have used the results of our calculations of energy levels [5], taking into account the closest pair of them. The other parameters are: $n_{i}=1 / a_{0}^{2}\left(a_{0}=5 \times 10^{-8} \mathrm{~cm}\right)$, $d=a_{0}, J=1 \cdot a_{0}^{3} \mathrm{eV} \cdot \mathrm{cm}^{3}$. For the halfwidth of energy gap outside the well we take the value $\Delta_{1}=0.8 \mathrm{eV}$ which corresponds to the case of EuS semiconductor. The dependence of interaction $E_{\text {int }}$ on the well width $L$ is presented in Fig. 2 for different values of $\Delta_{0}$ (halfwidth of the energy gap inside the quantum well).

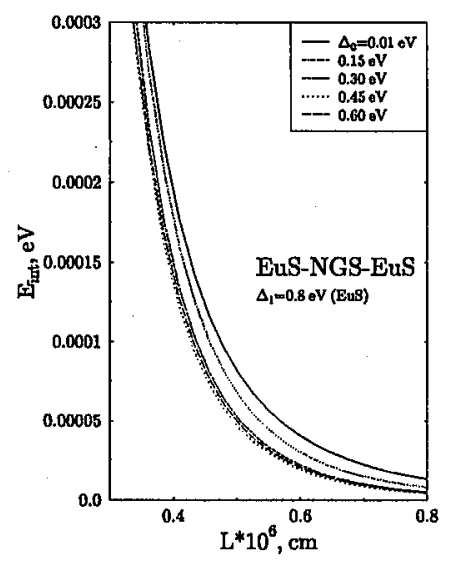

Fig. 2. Interaction energy vs. QW width for different values of the energy gap.

When there are magnetic impurities within a quantum well separating magnetic layers, an additional contribution to the layer coupling can appear. We assume that the magnetic impurities are distributed homogeneously inside the quantum well with a volume density $N_{i}$. Then after calculating the contribution of all homogenously distributed magnetic impurities, we get

$$
E_{\mathrm{int}}^{\mathrm{imp}}=-N_{i} J_{\mathrm{s}}(L) J \frac{N_{v} E_{m}}{4 v^{2}}\left\{\begin{array}{cc}
S_{z}^{(i)} \cdot S_{z}, & \boldsymbol{S}^{(i)}, \boldsymbol{S} \| z, \\
\frac{1}{4} S_{\alpha}^{(i)} \cdot S_{\alpha}, & \boldsymbol{S}^{(i)}, \boldsymbol{S} \perp z .
\end{array}\right.
$$


The results of numerical calculations show that the contribution of magnetic impurities is rather small if their density is smaller than $10^{18} \mathrm{~cm}^{-3}$ (in other words, unless $\mathrm{PbS}$ layer is purposely doped with magnetic impurities).

We presented here the results of calculations based on a simple energy model of the narrow-gap semiconducting heterostructure. In reality, several complications may arise which may modify the numerical values for magnetic coupling.

First, a usual uncertainty exists about the band offset between constituents of the heterostructure. It was the main reason for us to adopt the simplest energy model with symmetrical bands (type I QW). We believe that any other complications related with the initial model of the IV-VI narrow-gap energy spectrum, including the possible role of stresses at the interface, or deviation of the well shape from rectangular, are at the same footing. It is known, however, that for $\mathrm{EuS} / \mathrm{PbS}$ structure, there exists nearly perfect lattices matching [13], whereas the $\mathrm{X}$-ray measurements suggest the sharp EuS/PbS interface [6]. Secondly, there may exist a contribution to the coupling from real carriers (electrons and/or holes). In case of strongly degenerate electron (hole) gas inside the well, the corresponding contribution is the same as for the usual metal $[1,2]$. The presented here results correspond to the case of undoped material inside the well. In the experiments with $\mathrm{EuS} / \mathrm{PbS}$ structure [6] it has been reported that the carrier density in $\mathrm{PbS}$ is rather low.

This work was supported in part by the Committee for Scientific Research (Poland) under grant 2 PO3B 109 12, and by STCU (Ukraine) under grant 591.

\section{References}

[1] P. Bruno, Phys. Rev. B 52, 411 (1995).

[2] P. Bruno, Acta Phys. Pol. A 91, 37 (1997).

[3] M.D. Stiles, Phys. Rev. B 48, 7238 (1993).

[4] J. Blinowski, P. Kacman, Acta Phys. Pol. A 92, 719 (1997).

[5] V.K. Dugaev, P.P. Petrov, Phys. Status. Solidi B 184, 347 (1994).

[6] A. Stachow-Wójcik, A. Twardowski, T. Story, W. Dobrowolski, E. Grodzicka, A. Sipatow, Acta Phys. Pol. A 92, 985 (1997).

[7] V.K. Dugaev, V.I. Litvinov, P.P. Petrov, Superlattices Microstruct. 16, 413 (1994).

[8] V.K. Dugaev, V.I. Litvinov, P.P. Petrov, Semicond. Sci. Technol. 11, 80 (1996).

[9] N. Bloembergen, T. Rowland, Phys. Rev. 97, 1679 (1955).

[10] K.H. Herrmann, J. Auth, K.-P. Moellmann, J.W. Tomm, H. Boettner, A. Lambrecht, M. Tacke, I.V. Kolesnikov, A.E. Yunovich, A.I. Fedorenko, O.A. Mironov, A.Yu. Sipatov, Semicond. Sci. Technol. 8, S176 (1993).

[11] V.K. Dugaev, V.I. Litvinov, W. Dobrowolski, T. Story, Solid State Commun. 110, 351 (1999).

[12] G. Nimtz, B. Schlicht, in: Narrow-Gap Semiconductors, Springer, Berlin 1985, p. 1 .

[13] I.V. Kolesnikov, A.Yu. Sipatov, Sov. Phys.-Semicond. 26, 598 (1989). 